\title{
Optimization based stabilization of sampled-data nonlinear systems via their approximate discrete-time models
}

\author{
Lars Grüne \\ Fachbereich Mathematik \\ J.W. Goethe-Universität \\ Postfach 111932 \\ 60054 Frankfurt a.M., Germany \\ gruene@math.uni-frankfurt.de
}

\author{
Dragan Nešić \\ Department of Electrical \\ and Electronic Engineering \\ The University of Melbourne \\ Victoria 3010, Australia \\ d. nesic@ee.mu.oz.au
}

February 12, 2002

\begin{abstract}
We present results on numerical regulator design for sampled-data nonlinear plants via their approximate discrete-time plant models. The regulator design is based on an approximate discrete-time plant model and is carried out either via an infinite horizon optimization problem or via a finite horizon with terminal cost optimization problem. In both cases we discuss situations when the sampling period $T$ and the integration period $h$ used in obtaining the approximate discretetime plant model are the same or they are independent of each other. We show that using this approach practical and/or semiglobal stability of the exact discrete-time model is achieved under appropriate conditions.
\end{abstract}

\section{AMS Classification:}

Keywords: Controller design, asymptotic controllability, stabilization, numerical methods, optimal control.

\section{Introduction}

Stabilization of controlled systems is one of the central topics in control theory that has lead to a wealth of different stabilization techniques. An important set of stabilization methods is based on optimization techniques, such as receding horizon control (RHC) or model predictive control (MPC) (see [14,7] and references defined therein). In optimization based stabilization methods one can either compute control signals on-line, like in MPC algorithms, or off-line, like in $[8,9,13]$. In either case, it is common to implement the controller using a computer with $\mathrm{A} / \mathrm{D}$ and $\mathrm{D} / \mathrm{A}$ converters (sampler and zero-order hold) which leads to investigation of sampled-data nonlinear systems.

One of the main issues in sampled-data nonlinear control is the fact that the control designer usually can not compute the exact discrete-time model of the plant and has to use an approximate discrete-time model when designing a stabilizing controller. The approximate model is obtained by numerically integrating the continuous-time plant dynamics over 
one sampling interval while keeping the control constant (if a zero order hold is used). However, it is typically assumed in the optimization based stabilization literature that the exact discrete-time plant model is available for controller design (see for instance [6, 14, $13,12,11,1])$. Hence, there are gaps in the literature between the developed theory that is based on exact discrete-time models and the actual implementation of algorithms that invariably make use of approximate discrete-time models to compute control actions (see Example 1 in [3], Section V in [6] and Section IV in [13]). It is the purpose of this paper to present a careful investigation of the effects that numerical errors in approximatind the model may have on the stabilization of the exact discrete-time model.

While it may seem that any controller that stabilizes a sufficiently "good" approximate model would always stabilize the exact model for sufficiently small values of integration and/or sampling period, this issue is much more subtle than it may appear at a first glance. Indeed, a number of counter-examples illustrating different mechanisms that cause instability of exact models when controlled by controllers that stabilize approximate models have been presented in $[15,17]$. Moreover, results in $[15,17]$ present a set of general sufficient conditions on the continuous-time plant model, approximate discrete-time plant model and the designed controller that guarantee that controllers that stabilize the approximate model would also stabilize the exact model for sufficiently small sampling and/or integration period. Moreover, backstepping results in [16] show that controller design within the framework proposed in [15] may lead to considerable performance improvement as opposed to controller design based on continuous-time plant model that is followed by discretization of the controller (emulation design).

Results in $[15,17]$ present a framework for controller design via approximate discrete-time models but they do not explain how the actual controller design can be carried out within this framework. It is the purpose of this paper to investigate several situations when the optimization based stabilization is done within the framework of $[15,17]$. In particular, we consider the following problem:

Suppose we are given a family of approximate discrete-time plant models

$$
x(k+1)=F_{T, h}^{a}(x(k), u(k)),
$$

that are parameterized with the sampling period $T$ and a modeling parameter $h$, which is typically the integration period of the underlying integration scheme. Given a family of cost functions $J_{T, h}$, suppose that a family of controllers

$$
u(k)=u_{T, h}^{a, *}(x(k)),
$$

minimizes the given family of costs and is stabilizing for the family of approximate models. When would the same family of controllers stabilize the family of exact models

$$
x(k+1)=F_{T, h}^{e}(x(k), u(k)),
$$

for sufficiently small values of the modeling parameter $h$ ? 
We present conditions that guarantee that the family of controllers $u_{T, h}^{a, *}$ stabilizes in an appropriate sense the family of exact models for sufficiently small values of the modeling parameter. Two important situations are considered:

(i) $J_{T, h}$ is an infinite horizon cost function;

(ii) $J_{T, h}$ is a finite horizon cost function with a terminal cost.

In either case, we discuss two important sub-cases:

(i) $T$ and $h$ are independent of each other. This case is important when the sampling period $T$ is fixed and the family of approximate models is generated via a numerical integration method with adjustable integration step $h$. This case usually produces better results but the numerical computations required are more intensive (see, for instance, $[13,6])$.

(ii) $T=h$ and $T$ can be arbitrarily adjusted. This case is often used in the literature. The main motivation for using this approach is lessened computational burden in obtaining the approximate model but it will will be shown below that this method requires much stronger conditions than the first method (see [3]).

While our results do not cover all possible costs $J_{T, h}$ of interest, the presented proofs can be adapted to cover many other important situations. Moreover, the results we present are important in cases when the approximation of the plant model comes from a completely different mechanism than numerical integration of the plant dynamics. For example, the modeling parameter $h$ may capture the size of the cells used in the space discretization that is usually needed in numerical calculation of the controller via optimization techniques, such as dynamic programming (see [13]). The modeling parameter $h$ can be in general a vector capturing several different approximation mechanisms in obtaining the plant model and our results can be extended to cover this important case.

The paper is organized as follows. In Section 2 we present several motivating examples. Preliminaries are presented in Section 3. Several results from $[15,17]$ that we use to prove our main results are presented in Section 4. Infinite horizon and finite horizon optimization based stabilization problems are respectively considered in Sections 5 and 6 . Conclusions are presented in the last section and some auxiliary Lemmas are stated and proved in the Appendix.

\section{Motivation}

In this section we present two examples for which a family of optimal control laws is designed to stabilize the family of approximate models but the exact discrete-time model is destabilized for sufficiently fast sampling by the same family of controllers. These examples strongly motivate results of our paper.

Example 2.1 We consider the sampled data control of the triple integrator (this example was taken from [17])

$$
\dot{x}_{1}=x_{2}, \quad \dot{x}_{2}=x_{3}, \quad \dot{x}_{3}=u .
$$


While the exact discrete-time model of this system can be computed, we base our control algorithm on the family of Euler approximate discrete-time models in order to illustrate possible pitfalls in optimal control design based on approximate discrete-time models. The family of Euler approximate discrete-time models is

$$
\begin{aligned}
& x_{1}(k+1)=x_{1}(k)+T x_{2}(k) \\
& x_{2}(k+1)=x_{2}(k)+T x_{3}(k) \\
& x_{3}(k+1)=x_{3}(k)+T u(k) .
\end{aligned}
$$

Denote $x_{i}:=x_{i}(0), i=1,2,3, x:=\left(x_{1} x_{2} x_{3}\right)^{T}$ and $\bar{u}:=\{u(0), u(1), u(2), \ldots\}$. A minimum time dead beat controller for the Euler discrete-time model is designed to minimize the cost

$$
\begin{aligned}
J_{T}(x, \bar{u})= & x^{T}(3) x(3)=\left(x_{1}+3 T x_{2}+3 T^{2} x_{3}+T^{3} u(0)\right)^{2} \\
& +\left(x_{2}+3 T x_{3}+2 T^{2} u(0)+T^{2} u(1)\right)^{2}+\left(x_{3}+T u(0)+T u(1)+T u(2)\right)^{2}
\end{aligned}
$$

and we obtain the optimal controller

$$
u_{T}^{*}(x)=\left(-\frac{x_{1}}{T^{3}}-\frac{3 x_{2}}{T^{2}}-\frac{3 x_{3}}{T}\right) .
$$

The closed loop system (2.1)-(2.2) has all poles equal to zero for all $T>0$ and hence this discrete-time Euler-based closed loop system is asymptotically stable for all $T>0$. On the other hand, the closed loop system consisting of the exact discrete-time model of the triple integrator and the optimal controller $(2.2)$ has a pole at $\approx-2.644$ for all $T>0$. Hence, the optimal controller for approximate model destabilizes the exact model for any sampling period.

Example 2.2 Consider the scalar linear system:

$$
\dot{x}=x+u,
$$

whose exact discrete-time model is

$$
x(k+1)=e^{T} x(k)+\left(e^{T}-1\right) u(k) .
$$

We use the Euler model

$$
x(k+1)=(1+T) x(k)+T u(k)=F_{T} x(k)+G_{T} u(k)
$$

for controller design. Consider the following cost

$$
J_{T}(x, \bar{u})=\sum_{k=0}^{\infty}\left(Q_{T} x^{2}(k)+R_{T} u^{2}(k)\right),
$$

where $Q_{T}=T$ and $R_{T}=T^{3}(1-T)^{3}$. Obviously, the instantaneous cost $Q_{T} x^{2}+R_{T} u^{2}$ is a positive definite function of $x, u$ for all $T \in(0,1)$. Using [2, p. 53-54] we can obtain the family of optimal controllers for (2.5) as

$$
u_{T}^{*}(x)=\frac{G_{T} F_{T} S_{T}}{G_{T}^{2} S_{T}+R_{T}} x
$$


where $S_{T}$ is the solution of the following Ricatti equation

$$
S_{T}=F_{T}^{2}\left(S_{T}-\frac{S_{T}^{2} G_{T}^{2}}{G_{T}^{2} S_{T}+R_{T}}\right)+Q_{T} .
$$

Using the computer algebra system MAPLE, we computed the family of optimal control laws to be

$$
u_{T}^{*}(x)=\left(-1-\frac{5}{2} T^{2}+O\left(T^{3}\right)\right) x,
$$

which for sufficiently small $T$ yields stable approximate closed loop

$$
x(k+1)=\left(1-\frac{5}{2} T^{3}+O\left(T^{4}\right)\right) x(k) .
$$

However, the same family of controllers yields unstable exact closed loop

$$
x(k+1)=\left(1+\frac{1}{2} T^{2}+O\left(T^{3}\right)\right) x(k)
$$

for all sufficiently small $T$. Again the family of optimal controllers for the family of approximate models is destabilizing for the family of exact models for all sufficiently small sampling periods $T$.

Remark 2.3 Note that the optimal controller gain in the first example is not uniformly bounded in $T$ and in particular as $T \rightarrow 0$ we have for any $x \neq 0$ that $\left|u_{T}(x)\right| \rightarrow \infty$. It may appear that this is the only reason why instability of the exact model occurs. However, in the second example we have that the optimal controller gain is bounded uniformly in $T$ and yet instability occurs. More similar examples that do not use optimal control laws can be found in [17].

In both above examples we can say that the used cost $J_{T}(x, \bar{u})$ is ill parameterized with $T$ and this causes instability of the exact closed loop. In the sequel, we present conditions for well parameterized costs that avoid problems presented in the examples.

Remark 2.4 The interpretation of the above results is as follows. One can not first find a sufficiently "good" approximate plant model with a sufficiently small sampling and/or integration period and then assume that the optimal controller for the approximate model with respect to any given cost would stabilize the exact model. Indeed, because of the fact that we are considering parameterized systems and costs, the examples illustrate that given arbitrarily small sampling period (and hence arbitrarily "good" plant model) there exists a cost function for which the controller that is optimal for the approximate model would destabilize the exact model. Hence, a careful investigation of stability is needed to avoid situations presented in examples.

\section{Preliminaries}

$\mathbb{R}$ and $\mathbb{N}$ denote respectively the sets of real and natural numbers. We also denote $\mathbb{N}_{0}:=$ $\{0\} \cup \mathbb{N}$. In the Euclidean space $\mathbb{R}^{n},\|\cdot\|$ denotes the usual Euclidean norm and $B_{r}$ and $\bar{B}_{r}$ 
denote respectively the open and closed ball with radius $r$ around the origin. A continuous function $\gamma: \mathbb{R}_{0}^{+} \rightarrow \mathbb{R}_{0}^{+}$is called of class $\mathcal{K}$ if it is strictly increasing with $\gamma(0)=0$; it is called of class $\mathcal{K}_{\infty}$ if it is of class $\mathcal{K}$ and unbounded. A continuous function $\beta: \mathbb{R}_{0}^{+} \times \mathbb{R}_{0}^{+} \rightarrow \mathbb{R}_{0}^{+}$ is called of class $\mathcal{K} \mathcal{L}$ if it is of class $\mathcal{K}$ in the first argument and strictly decreasing to 0 in the second.

Consider a continuous-time plant given by

$$
\dot{x}=f(x, u),
$$

where $x \in \mathbb{R}^{n}$ and $u \in U \subseteq \mathbb{R}^{m}$ with $0 \in U$. The plant is to be controlled via a computer that is connected to the plant via a sampler and zero order hold. We assume that $f$ is locally Lipschitz, which guarantees that the solutions of (3.1) exist locally in time. Let $\phi\left(t, x_{0}, u\right)$ denote the solution trajectory for time $t$, initial value $x$ and constant control function $u \in U$. Suppose that for a given $T, x, u$ the solution $\phi(t, x, u)$ exists for all $t \in[0, T]$. Then, we can introduce the exact discrete-time model of the system

$$
x_{k+1}=F_{T}^{e}(x(k), u(k)),
$$

where $F_{T}^{e}(x, u):=\phi(T, x, u)$. Note that the trajectories of (3.1) may have finite escape time, in which case $F_{T}^{e}(x, u)$ might not be defined for all $x \in \mathbb{R}^{n}, u \in U$. However, since $f$ is assumed locally Lipschitz we have that for each $\Delta>0$ there exists $T_{\Delta}^{*}>0$ such that $F_{T}^{e}(x, u)$ exists for all $x \in \bar{B}_{\Delta}, u \in \bar{B}_{\Delta}$ and all $T \in\left(0, T_{\Delta}^{*}\right]$. The set of all control sequences is denoted by $\mathcal{U}$, members of $\mathcal{U}$ will be denoted by $\bar{u}=(u(k))_{k \in \mathbb{N}_{0}}$.

We note that since $f$ is typically nonlinear, $F_{T}^{e}$ in (3.2) is not known in most cases. Hence, if we want to carry out controller design for the sampled-data plant (3.1) via its discrete-time model, we need to use an approximate discrete-time model instead

$$
x_{k+1}=F_{T, h}^{a}(x(k), u(k))
$$

where $T \in\left(0, T^{*}\right]$ is the sampling rate with some upper bound $T^{*}>0$ and $h \in(0, T]$ is a parameter for the accuracy of the approximate model, e.g., the integration step for some underlying numerical one-step approximation.

Remark 3.1 The map $F_{T, h}^{a}$ defining the approximate model is typically interpreted as a numerical approximation of $F_{T}^{e}$ using some suitable numerical scheme. For instance, $F_{T, h}^{a}$ might be constructed using multiple steps of a one-step Runge-Kutta scheme $\Phi_{h_{i}}$ with integration step sizes $h_{i}, i=1, \ldots, m$ satisfying $h_{i} \leq h$ and $\sum_{i=1}^{m} h_{i}=T$, i.e.,

$$
x_{\circ}=x, x_{i+1}=\Phi_{h_{i}}\left(x_{i}, u\right), \quad F_{T, h}^{a}(x, u)=x_{m} .
$$

Note that for constant control functions $u$ system (3.1) is an autonomous ODE, hence all numerical schemes for autonomous ODEs are applicable, see, e.g., [10], [21] or [22] for a description of suitable numerical methods. In the simplest case, $\Phi_{h_{i}}$ could be chosen as the Euler method $\Phi_{h_{i}}(x, u)=x+h f(x, u)$. Note that any $T, h$ the numerical scheme $F_{T, h}^{a}(x, u)$ will normally exist for all $x, u$ because the computation of $F_{T, h}^{a}$ is usually based on finitely many evaluations of $f$ only. 
Given a family of cost functions $J_{T, h}(x, \bar{u})$ we will design a family of optimal control law for the approximate model

$$
u(k)=u_{T, h}^{a, *}(x(k)),
$$

and investigate when they stabilize the family of exact models (3.2) for all small $h$.

In general, it is useful to consider exact models that are also parameterized by a modeling parameter (for motivation see [17])

$$
x_{k+1}=F_{T, h}^{e}(x(k), u(k)) .
$$

In this case, however, $h$ is not interpreted as a numerical integration step. We write $F_{T, h}$ if we refer to a general discrete-time parameterized system

$$
x_{k+1}=F_{T, h}(x(k), u(k)),
$$

in particular, $F_{T, h}$ may stand for both $F_{T, h}^{e}$ and $F_{T, h}^{a}$. The special case $T=h$ has received a lots attention in the literature and in this case we will write $F_{T}$ instead of $F_{T, T}$. Given $\bar{u}$ and $x_{\circ}$, the trajectories of the systems (3.5) and (3.3) are denoted respectively by $\phi_{T, h}^{e}\left(k, x_{\circ}, \bar{u}\right)$ and $\phi_{T, h}^{a}\left(k, x_{\circ}, \bar{u}\right)$. Again, if we refer to a generic system (3.6) we use the notation $\phi_{T, h}\left(k, x_{\circ}, \bar{u}\right)$ and if $T=h$ we write $\phi_{T}$ instead of $\phi_{T, T}$.

Assumption 3.2 We assume that both $F_{T, h}^{e}$ and $F_{T, h}^{a}$ are continuous in $u$ and satisfy a local Lipschitz condition of the following type: for each $\Delta>0$ there exist $T>0, L>0$ and $h^{*}>0$ such that

$$
\left\|F_{T, h}(x, u)-F_{T, h}(y, u)\right\| \leq e^{L T}\|x-y\|
$$

holds for all $u \in \bar{B}_{\Delta}$ all $h \in\left(0, h^{*}\right]$ and all $x, y \in \bar{B}_{\Delta}$.

For the exact model this property is easily verified using Gronwall's Lemma (if $F_{T, h}^{e}$ is well defined), while for the approximate model it depends on the properties of the numerical scheme in use. For Runge-Kutta schemes, e.g., it is verified by induction using the property $\left\|\Phi_{h_{i}}(x, u)-\Phi_{h_{i}}(y, u)\right\| \leq\left(1+L h_{i}\right)\|x-y\|$, cf. [22], and the inequality $1+L h_{i} \leq e^{L h_{i}}$.

\section{Definitions and background results}

In $[15,17]$ sufficient conditions based on the Lyapunov second method were presented that guarantee that the family of controllers that stabilizes (3.3) would also stabilize (3.5) for sufficiently small $h$. Here the control laws under consideration do not need to come from optimal control problems, however, they will still be parametrized by the parameters $T$ and $h$. The results in this section will be used in the rest of this paper. In order to state these results we need several definitions.

Definition 4.1 Let strictly positive real numbers $\left(T, \Delta_{1}, \Delta_{2}\right)$ be given. If there exists $h^{*}>0$ such that

$$
\sup _{\left\{x \in B_{\Delta_{1}}, h \in\left(0, h^{*}\right]\right\}}\left|u_{T, h}(x)\right| \leq \Delta_{2}
$$


then we say that the family of controllers (3.4) is $\left(T, \Delta_{1}, \Delta_{2}\right)$-uniformly bounded. Moreover, if $T=h$ and if for any strictly positive $\Delta_{1}$ there exist strictly positive $\left(\Delta_{2}, h^{*}\right)$ so that (4.1) holds, then we say that the family of controllers (3.4) is semiglobally uniformly bounded.

The following "consistency" property is central in our developments and it is an appropriate adaptation and generalization of a consistency property used in the numerical analysis literature (see [22]):

Definition 4.2 Let a triple of strictly positive numbers $\left(T, \Delta_{1}, \Delta_{2}\right)$ be given and suppose that there exists $\gamma \in \mathcal{K}$ and $h^{*}>0$ such that

$$
(x, u) \in B_{\Delta_{1}} \times B_{\Delta_{2}}, h \in\left(0, h^{*}\right] \Longrightarrow\left\|F_{T, h}^{a}(x, u)-F_{T, h}^{e}(x, u)\right\| \leq T \gamma(h)
$$

Then we say that the family $F_{T, h}^{a}$ is $\left(T, \Delta_{1}, \Delta_{2}\right)$-consistent with $F_{T, h}^{e}$. Moreover, if $T=h$ and if for any pair of strictly positive numbers $\left(\Delta_{1}, \Delta_{2}\right)$ there exist $\gamma \in \mathcal{K}$ and $h^{*}>0 \mathrm{such}$ that (4.2) holds, then we say that $F_{T, h}^{a}$ is semiglobally consistent with $F_{T, h}^{e}$.

Sufficient checkable conditions for consistency properties can be found in $[15,17]$.

Definition 4.3 Let a pair of strictly positive real numbers $(T, D)$, a family of functions $V_{T, h}: \mathbb{R}^{n} \rightarrow \mathbb{R}_{\geq 0}$, functions $\sigma_{1}, \sigma_{2} \in \mathcal{K}_{\infty}$ and a positive definite function $\sigma_{3}: \mathbb{R}_{\geq 0} \rightarrow \mathbb{R}_{\geq 0}$ be given. Suppose for any pair of strictly positive real numbers $\left(\delta_{1}, \delta_{2}\right)$ with $\delta_{2}<D$ there exist $h^{*}>0$ and $c>0$ such that for all $x \in B_{D}, h \in\left(0, h^{*}\right]$, we have

$$
\begin{gathered}
\sigma_{1}(\|x\|) \leq V_{T, h}(x) \leq \sigma_{2}(\|x\|) \\
V_{T, h}\left(F_{T, h}^{a}\left(x, u_{T, h}(x)\right)-V_{T, h}(x) \leq-T \sigma_{3}(\|x\|)+T \delta_{1},\right.
\end{gathered}
$$

and, for all $x_{1}, x_{2} \in B_{D}-B_{\delta_{2}}$, with $\left\|x_{1}-x_{2}\right\| \leq c$ we have

$$
\left|V_{T, h}\left(x_{1}\right)-V_{T, h}\left(x_{2}\right)\right| \leq \delta_{1} .
$$

Then we say that the family $(3.6),(3.4)$ is $(T, D)$-stable with a continuous Lyapunov function. Moreover, if $T=h$ and for any triple of strictly positive real numbers $\left(D, \delta_{1}, \delta_{2}\right)$ with $\delta_{2}<D$ there exist $h^{*}>0$ and $L>0$ such that for all $x, x_{1}, x_{2} \in B_{D}, h \in\left(0, h^{*}\right]$, we have that (4.3), (4.4) and

$$
\left|V_{T}\left(x_{1}\right)-V_{T}\left(x_{2}\right)\right| \leq L\left\|x_{1}-x_{2}\right\|
$$

hold, then we say that the family (3.6), (3.4) is semiglobally stable with a Lipschitz Lyapunov function.

The following two theorems from $[15,17]$ play a central role in our developments.

Theorem 4.4 Suppose that there exist a triple of strictly positive numbers $(T, D, M)$ such that 
(i) The family of closed loop systems $\left(F_{T, h}^{a}, u_{T, h}^{a}\right)$ is $(T, D)$-stable with a continuous Lyapunov function.

(ii) The family of controllers $u_{T, h}^{a}$ is $(T, D, M)$-uniformly bounded.

(iii) The family $F_{T, h}^{a}$ is $(T, D, M)$-consistent with $F_{T, h}^{e}$.

Then, there exists $\beta \in \mathcal{K} \mathcal{L}, D_{1} \in(0, D)$ and for any $\delta>0$, there exists $h^{*}>0$ such that for all $x_{\circ} \in B_{D_{1}}$ and $h \in\left(0, h^{*}\right]$ the solutions of the family $\left(F_{T, h}^{e}, u_{T, h}^{a}\right)$ satisfy:

$$
\left\|\phi_{T, h}^{e}\left(k, x_{\circ}\right)\right\| \leq \beta\left(\left\|x_{\circ}\right\|, k T\right)+\delta, \quad \forall k \in \mathbb{N}_{0} .
$$

Theorem 4.5 Suppose that $T=h$ and the following conditions hold:

(i) The family of closed loop systems $\left(F_{T}^{a}, u_{T}^{a}\right)$ is semiglobally stable with a Lipschitz Lyapunov function.

(ii) The family of controllers $u_{T}^{a}$ is semiglobally uniformly bounded.

(iii) The family $F_{T}^{a}$ is semiglobally consistent with $F_{T}^{e}$.

Then, there exists $\beta \in \mathcal{K} \mathcal{L}$, such that for any $D_{1}>0$ and $\delta>0$, there exists $T^{*}>0$ such that for all $x_{\circ} \in B_{D_{1}}$ and $T \in\left(0, T^{*}\right]$ the solutions of the family $\left(F_{T}^{e}, u_{T}^{a}\right)$ satisfy:

$$
\left\|\phi_{T}^{e}\left(k, x_{\circ}\right)\right\| \leq \beta\left(\left\|x_{\circ}\right\|, k T\right)+\delta, \quad \forall k \in \mathbb{N}_{0} .
$$

Consistency can be checked using the properties of the approximate model (3.3) and continuous-time plant model (3.1). Hence, Theorems 4.4 and 4.5 provide general conditions on the controller, approximate model and continuous-time plant that guarantee that the controllers that are designed via approximate model would also stabilize exact model for sufficiently values of small modeling parameter. In the sequel we investigate the conditions under which control laws that are optimal in some sense for the approximate satisfy all conditions of Theorems 4.4 and 4.5 .

\section{Infinite horizon problems}

In the first part of this section we assume that $T \neq h$ and $h$ can be assigned arbitrarily and independently of $T$, which is arbitrary but fixed. In the second part we consider the case when $T=h$ and $T$ can be assigned arbitrarily.

\subsection{Stabilization with a fixed sampling rate $T$}

We consider the optimal control problem

$$
\min _{\bar{u} \in \mathcal{U}} \sum_{k=0}^{\infty} T l_{h}\left(\phi_{T, h}(k, x, \bar{u}), u(k)\right)
$$

where the running cost $l_{h}$ satisfies the following assumption. 
Assumption 5.1 The following hold:

(i) $l_{h}$ is continuous with respect to $x$ and $u$, uniformly in small $h$.

(ii) There exist $h^{*}>0$ and two class $\mathcal{K}_{\infty}$ functions $\rho_{1}$ and $\rho_{2}$ such that the inequality

$$
\rho_{1}(\|x\|+\|u\|) \leq l_{h}(x, u) \leq \rho_{2}(\|x\|+\|u\|)
$$

holds for all $x, u$ and $h \in\left(0, h^{*}\right]$.

(iii) For each $\Delta>0$ there exists $N>0$ and $h^{*}>0$ such that

$$
\left|l_{h}(x, u)-l_{h}(y, u)\right| \leq N\|x-y\|
$$

for all $h \in\left(0, h^{*}\right], x, y \in \mathbb{R}^{n}$ and all $u \in U$ with $\|x\|,\|y\|,\|u\| \leq \Delta$.

Note that the sum in (5.1) may diverge, hence it may take the value $\infty$. We make the convention that this sum takes the value $\infty$ if the trajectory $\phi_{T, h}(\cdot, x, \bar{u})$ does not exist for some $k \in \mathbb{N}_{0}$.

We denote the optimal cost functions related to the exact and the approximate system by

$$
W_{T, h}^{e}(x):=\min _{\bar{u} \in \mathcal{U}} \sum_{k=0}^{\infty} T l_{h}\left(\phi_{T, h}^{e}(k, x, \bar{u}), u(k)\right), \quad W_{T, h}^{a}(x):=\min _{\bar{u} \in \mathcal{U}} \sum_{k=0}^{\infty} T l_{h}\left(\phi_{T, h}^{a}(k, x, \bar{u}), u(k)\right)
$$

again using $W_{T, h}(x)$ if we want to refer to a general system and $W_{T}$ if $T=h$. Note that $W_{T, h}(x)=\infty$ is possible, so we will have to formulate conditions such that $W_{T, h}$ is finite at least for compact subsets of the state space.

It is clear that not every plant would allow for a meaningful solution of the optimal control problem (5.1). However, if the plant model satisfies the following asymptotic controllability assumption we will prove in Theorem 5.4 below that a solution to (5.1) exists under certain assumptions.

Definition 5.2 Let $T>0, \beta \in \mathcal{K} \mathcal{L}$ and $\Delta>0$ be given. The family of systems (3.6) is called $(T, \Delta, \beta)$-asymptotically controllable to the origin with vanishing controls if there exists $h^{*}>0$ such that for all $h \in\left(0, h^{*}\right]$ and each $x \in \bar{B}_{\Delta}$ there exists $\bar{u} \in \mathcal{U}$ such that

$$
\left\|\phi_{T, h}(k, x, \bar{u})\right\|+\|u(k)\| \leq \beta(\|x\|, T k), \quad k \in \mathbb{N}_{0} .
$$

Asymptotic controllability has been introduced in [19] and we have adapted the definition from [13] to be applicable to families of discrete-time systems. Note that this definition in particular requires $\|u(k)\| \leq \beta(\|x\|, T k)$. This assumption is mainly needed in order to simplify some of the following arguments and could be relaxed in various ways, e.g., to $\|u(k)\| \leq \delta+\beta(\|x\|, T k)$ for some $\delta>0$, provided that also Assumption 5.1 (ii) is suitably adjusted. The following result is used in the sequel. 
Proposition $5.3[20]$ Given an arbitrary $\beta \in \mathcal{K} \mathcal{L}$, there exist two functions $\alpha_{1}, \alpha_{2} \in \mathcal{K}_{\infty}$ such that the following holds:

$$
\beta(s, t) \leq \alpha_{1}\left(\alpha_{2}(s) e^{-t}\right) \quad \forall s, t \geq 0 .
$$

Note that using Proposition 5.3, there is no loss of generality if we assume that $\beta(s, t)$ in Definition 5.2 is replaced by $\alpha_{1}\left(\alpha_{2}(s) e^{-t}\right)$. The following theorem shows conditions under which the optimal feedback law for the approximate model exists and can be used to stabilize the exact closed loop system.

Theorem 5.4 Let strictly positive real numbers $(\Delta, T)$ and functions $\beta \in \mathcal{K} \mathcal{L}$ and $l_{h}(\cdot, \cdot)$ satisfying Assumption 5.1 be given. Let $\beta$ generate $\alpha_{1}, \alpha_{2} \in \mathcal{K}_{\infty}$ using Proposition 5.3 and let $l_{h}$ generate $\rho_{1}, \rho_{2} \in \mathcal{K}_{\infty}$ via (5.2). Suppose that:

(i) The family of approximate models $F_{T, h}^{a}$ satisfies Assumption 3.2.

(ii) The family of approximate models $F_{T, h}^{a}$ is $(T, \Delta, \beta)$-asymptotically controllable to the origin with vanishing controls.

(iii) There exists $C>0$ such that

$$
\int_{0}^{1} \frac{\rho_{2} \circ \alpha_{2}(s)}{s} d s \leq C
$$

Then, for the family of systems $F_{T, h}^{a}$ there exists a solution to the family of optimal control problems:

$$
\min _{\bar{u} \in \mathcal{U}} \sum_{k=0}^{\infty} T l_{h}\left(\phi_{T, h}^{a}(k, x, \bar{u}), u(k)\right),
$$

of the form

$$
u(k)=u_{T, h}^{a, *}(x(k)),
$$

and numbers $D \in(0, \Delta), M>0$ such that the following hold:

(i') The family of controllers $u_{T, h}^{a, *}$ is $(T, D, M)$-uniformly bounded.

(ii') The family $\left(F_{T, h}^{a}, u_{T, h}^{a, *}\right)$ is $(T, D)$-stable with continuous Lyapunov function.

Suppose, moreover, that the additional condition holds:

(iii') The family of approximate models $F_{T, h}^{a}$ is $(T, D, M)$-consistent with $F_{T, h}^{e}$.

Then, there exists $D_{1} \in(0, D)$ and $\beta_{1} \in \mathcal{K} \mathcal{L}$ and for any $\delta>0$ there exists $h^{*}>0$ such that for all $x_{\circ} \in B_{D_{1}}$ and all $h \in\left(0, h^{*}\right]$, the solutions of the family $\left(F_{T, h}^{e}, u_{T, h}^{a, *}\right)$ satisfy:

$$
\left\|\phi_{T, h}^{e}\left(k, x_{\circ}\right)\right\| \leq \beta_{1}\left(\left\|x_{\circ}\right\|, k T\right)+\delta, \quad \forall k \in \mathbb{N}_{0} .
$$


Proof: Let all the conditions of Theorem 5.4 be satisfied. First, we will prove that conditions (i), (ii) and (iii) imply conditions (i') and (ii'). Then, the last statement follows immediately from (i'), (ii') and (iii') via Theorem 4.4.

(i) $+($ ii $)+($ iii $) \Rightarrow\left(\right.$ ii' $\left.^{\prime}\right)$

We use the optimal value of the cost $W_{T, h}^{a}(x)$ as the Lyapunov function for the approximate closed loop system, which is standard in optimization literature. We now show that $W_{T, h}^{a}$ satisfies (4.3), (4.4) and (4.5) of Definition 4.3.

It is immediate from (5.2) that for any $x$ and $h \in\left(0, h^{*}\right]$ we have

$$
\sigma_{1}(\|x\|):=T \rho_{1}(\|x\|) \leq W_{T, h}^{a}(x) .
$$

Let $x \in \bar{B}_{\Delta}$ and $h \in\left(0, h^{*}\right]$. Using the definition of the cost, the bound (5.2) and condition (ii), we obtain for $\bar{u}$ from Definition 5.2

$$
\begin{aligned}
W_{T, h}^{a}(x) & \leq \sum_{k=0}^{\infty} T l_{h}\left(\phi_{T, h}^{a}(k, x, \bar{u}), u(k)\right) \\
& \leq \sum_{k=0}^{\infty} T \rho_{2}\left(\left\|\phi_{T, h}^{a}(k, x, \bar{u})\right\|+\|u(k)\|\right) \\
& \leq \sum_{k=0}^{\infty} T \rho_{2}(\beta(\|x\|, k T)) \\
& \leq \sum_{k=0}^{\infty} T \rho_{2}\left(\alpha_{2}\left(e^{-k T} \alpha_{1}(\|x\|)\right)\right) \\
& =T \rho_{2} \circ \alpha_{2} \circ \alpha_{1}(\|x\|)+\sum_{k=1}^{\infty} T \rho_{2}\left(\alpha_{2}\left(e^{-k T} \alpha_{1}(\|x\|)\right)\right) \\
& \leq T \rho_{2}\left(\alpha_{2}\left(\alpha_{1}(\|x\|)\right)\right)+\int_{0}^{\infty} \rho_{2}\left(\alpha_{2}\left(e^{-t} \alpha_{1}(\|x\|)\right)\right) d t
\end{aligned}
$$

It was shown in [4, Proof of Theorem 1] that under condition (iii) the integral term in the last inequality can be bounded by $\tilde{\sigma}(\|x\|)$ for some $\tilde{\sigma} \in \mathcal{K}_{\infty}$. Hence, if we define $\sigma_{2}(r):=T \rho_{2}\left(\alpha_{2}\left(\alpha_{1}(r)\right)\right)+\tilde{\sigma}(r)$, we can write for all $x \in \bar{B}_{\Delta}$ and $h \in\left(0, h^{*}\right]$ that:

$$
W_{T, h}^{a}(x) \leq \sigma_{2}(\|x\|)
$$

Hence, (5.6) and (5.7) show that (4.3) of Definition 4.3 holds.

Let an arbitrary $\delta_{1}>0$ be given. We show now that for the given $\left(\Delta, \delta_{1}\right)$ there exist $D \in(0, \Delta], c>0$ and $h^{*}>0$ such that the implication

$$
x \in \bar{B}_{D},\|x-y\| \leq c, h \in\left(0, h^{*}\right] \Rightarrow\left|W_{T, h}^{a}(x)-W_{T, h}^{a}(y)\right| \leq \delta_{1}
$$

holds, which proves that (4.5) is satisfied ${ }^{1}$.

\footnotetext{
${ }^{1}$ Note that this is a stronger condition than what is needed in Definition 4.3 since we have $\delta_{2}=0$.
} 
For the rest of the proof we use lemmas that are presented and proved in the appendix. Let $\rho_{1}, \rho_{2} \in \mathcal{K}_{\infty}$ and $h_{1}^{*}>0$ come from Assumption 5.1. Define the following numbers:

$$
\begin{aligned}
S & :=\sigma_{1}(\Delta)+\delta_{1} / 4 \\
\widetilde{\Delta} & :=\rho_{1}^{-1}(S / T) \\
\alpha & =\sigma_{2}^{-1}\left(\frac{\delta_{1}}{8}\right)
\end{aligned}
$$

Let $(S, \alpha / 2)$ generate via Lemma 8.2 the number $\tau>0$. Let $\widetilde{\Delta}$ generate via (3.7) the number $N>0$ and $h_{2}^{*}>0$. Let $(\tilde{\Delta}, \tau, T)$ and $\delta:=\min \left\{\alpha / 2, \frac{\delta_{1}}{2 N \tau}\right\}$ generate via Lemma 8.4 the numbers $c>0$ and $h_{3}^{*}>0$. Let $h^{*}:=\min \left\{h_{1}^{*}, h_{2}^{*}, h_{3}^{*}\right\}$. Let $D:=\sigma_{2}^{-1} \circ \sigma_{1}(\Delta)$.

In all calculations below we consider arbitrary $x \in \bar{B}_{D}, h \in\left(0, h^{*}\right]$ and $\|x-y\| \leq c$. Let $\bar{u}$ be a control sequence such that

$$
\sum_{k=0}^{\infty} l_{h}\left(\phi_{T, h}^{a}(k, x, \bar{u}), u(k)\right) \leq W_{T, h}^{a}(x)+\delta_{1} / 4,
$$

which implies from $\|x\| \leq D$ and the definition of $S$ that $\sum_{k=0}^{\infty} l_{h}\left(\phi_{T, h}(k, x, \bar{u}), u(k)\right) \leq S$. From Lemma 8.1 and the definition of $\widetilde{\Delta}$ we have

$$
\left\|\phi_{T, h}^{a}(k, x, \bar{u})\right\|+\|u(k)\| \leq \widetilde{\Delta} \quad \forall k \in \mathbb{N}_{0} .
$$

From the definition of $\alpha$ and (5.7) we have $W_{T, h}^{a}(x) \leq \delta_{1} / 8$ for all $x \in \bar{B}_{\alpha}$. From our choice of $\tau$ it follows from Lemma 8.2 that for some $j \in \mathbb{N}_{0}$ with $T j \leq \tau$ we have $\left\|\phi_{T, h}^{a}(j, x, u)\right\| \leq \alpha / 2$. Moreover, from Lemma 8.4 and our choice of $\delta$ it follows that $\left\|\phi_{T, h}^{a}(j, x, \bar{u})-\phi_{T, h}^{a}(j, y, \bar{u})\right\| \leq \delta \leq \alpha / 2$ and consequently $\left\|\phi_{T, h}^{a}(j, y, \bar{u})\right\| \leq \alpha$ which implies from the choice of $\alpha$ that

$$
W_{T, h}^{a}\left(\phi_{T, h}^{a}(j, y, \bar{u})\right) \leq \delta_{1} / 8
$$

Abbreviating $\tilde{y}=\phi_{T, h}^{a}(j, y, \bar{u})$ we can choose a control sequence $\bar{u}^{*}$ satisfying

$$
\sum_{k=0}^{\infty} l_{h}\left(\phi_{T, h}^{a}\left(k, \tilde{y}, \bar{u}^{*}\right), u^{*}(k)\right) \leq W_{T, h}^{a}(\tilde{y})+\delta_{1} / 8 \leq \delta_{1} / 4
$$

Replacing $u(k), k=j, j+1, \ldots$ by $u_{k-j}^{*}$ we thus obtain

$$
\begin{aligned}
W_{T, h}^{a}(y) & \leq \sum_{k=0}^{j-1} T l_{h}\left(\phi_{T, h}^{a}(k, y, \bar{u}), u(k)\right)+\sum_{k=j}^{\infty} T l_{h}\left(\phi_{T, h}^{a}(k, y, \bar{u}), u(k)\right) \\
& =\sum_{k=0}^{j-1} T l_{h}\left(\phi_{T, h}^{a}(k, y, \bar{u}), u(k)\right)+\sum_{k=0}^{\infty} T l_{h}\left(\phi_{T, h}^{a}\left(k, \tilde{y}, \bar{u}^{*}\right), u^{*}(k)\right) \\
& \leq \sum_{k=0}^{j-1} T l_{h}\left(\phi_{T, h}^{a}(k, y, \bar{u}), u(k)\right)+\delta_{1} / 4 .
\end{aligned}
$$


Again using Lemma 8.4, the Lipschitz property of $l_{h}$, the fact that $j T \leq \tau$ and our choice of $\delta$ we can conclude that

$$
\sum_{k=0}^{j-1} T\left(l_{h}\left(\phi_{T, h}^{a}(k, y, \bar{u}), u(k)\right)-l_{h}\left(\phi_{T, h}^{a}(k, x, \bar{u}), u(k)\right)\right) \leq N \tau \delta \leq \delta_{1} / 2 .
$$

The definition of $W_{T, h}^{a}$, the choice of $\bar{u}$ and the positive definiteness of $l_{h}$ imply

$$
W_{T, h}^{a}(x) \geq \sum_{k=0}^{j-1} T l_{h}\left(\phi_{T, h}^{a}(k, x, \bar{u})\right)-\delta_{1} / 4 .
$$

We now combine (5.9), (5.10) and (5.11)

$$
\begin{aligned}
W_{T, h}^{a}(y)-W_{T, h}^{a}(x) \leq & \sum_{k=0}^{j-1} T l_{h}\left(\phi_{T, h}^{a}(k, y, \bar{u}), u(k)\right) \\
& \quad-\sum_{k=0}^{j-1} T l_{h}\left(\phi_{T, h}^{a}(k, x, \bar{u}), u(k)\right)+\delta_{1} / 4+\delta_{1} / 4 \\
& \leq \delta_{1} / 2+\delta_{1} / 4+\delta_{1} / 4=\delta_{1} .
\end{aligned}
$$

Since the corresponding estimate for $W_{T}^{a}(x)-W_{T}^{a}(y)$ follows by symmetry, this completes the proof of (4.5).

Finally, with the given $\left(\Delta, \delta_{1}\right)$ we show that (4.4) is satisfied. For any fixed $T$ and $h$, standard optimal control arguments show that $W_{T, h}^{a}$ satisfies the dynamic programming equation

$$
W_{T, h}^{a}(x)=\inf _{u \in U}\left\{T l_{h}(x, u)+W_{T, h}^{a}\left(F_{T, h}^{a}(x, u)\right)\right\} .
$$

Since $F_{T, h}^{a}$ and $l_{h}$ are continuous in $u, W_{T, h}^{a}$ is continuous in $x$ and $l_{h}$ is positive definite the "inf" is actually a "min" and we can define the desired $u_{T, h}^{a, *}(x)$ by choosing it such that

$$
T l_{h}\left(x, u_{T, h}^{a, *}(x)\right)+W_{T, h}^{a}\left(F_{T, h}^{a}\left(x, u_{T, h}^{a, *}(x)\right)\right)=\min _{u \in U}\left\{T l_{h}(x, u)+W_{T, h}^{a}\left(F_{T, h}^{a}(x, u)\right)\right\} .
$$

Combining the above given bounds and using (5.2), we obtain

$$
\begin{aligned}
W_{T, h}^{a}\left(F_{T, h}^{a}\left(x, u_{T, h}^{a, *}(x)\right)\right)-W_{T, h}^{a}(x) & =-T l_{h}\left(x, u_{T, h}^{a, *}(x)\right) \\
& \leq-T \rho_{1}(\|x\|) \\
& \leq-T \rho_{1}(\|x\|)+T \delta_{1},
\end{aligned}
$$

which proves (4.4) and completes the proof of (ii').

(i) $+($ ii $)+($ iii $) \Rightarrow\left(\right.$ (i') $^{\prime}$

Since for all $x \in \bar{B}_{D}$ we have $T l_{h}\left(x, u_{T, h}^{a, *}(x)\right) \leq W_{T, h}^{a}(x)$ and since (5.2) holds, we can write that

$$
\begin{aligned}
\left\|u_{T, h}^{a, *}(x)\right\| & \leq \rho_{1}^{-1}\left(l_{h}\left(x, u_{T, h}^{a, *}(x)\right)\right) \\
& \leq \rho_{1}^{-1}\left(\frac{1}{T} W_{T, h}^{a}(x)\right) \\
& \leq \rho_{1}^{-1}\left(\frac{1}{T} \sigma_{2}(\|x\|)\right) \\
& \leq \rho_{1}^{-1}\left(\frac{\sigma_{2}(D)}{T}\right)=: M,
\end{aligned}
$$


which proves that (i') holds.

Remark 5.5 Note that if $T$ can be adjusted arbitrarily and independent of $h$, and, moreover, for any arbitrary $\Delta>0$ there exists $T$ so that the system is $(T, \Delta, \beta)$-asymptotically controllable with vanishing controls, and all other conditions of Theorem 5.4 hold, then all conclusions of Theorem 5.4 hold. Hence, for $T$ varying and independent of $h$ we can modify the statement of Theorem 5.4 to obtain a result on semiglobal practical stabilization. However, if $T=h$, we need much stronger conditions to achieve semiglobal practical stabilization which is discussed in more detail in the next subsection.

Remark 5.6 Neither of the examples of Section 2 satisfies Assumption 5.1 and that is the reason why the controllers $u_{T}^{a, *}$ do not stabilize the family of exact models $F_{T}^{e}$.

Remark 5.7 It is possible under mild conditions to obtain $\mathcal{K} \mathcal{L}$ stability bounds for the solutions of the sampled-data system from the $\mathcal{K} \mathcal{L}$ stability bounds for the exact discretetime model and bounds on the inter-sample behavior, as illustrated in [18].

\subsection{Stabilization with varying sampling rate $T=h$}

The case when $T=h$ is sometimes considered in the literature (see Example 1 in [3]) and we discuss it next. For instance, some authors use the Euler approximate model

$$
x(k+1)=F_{T}^{a}(x(k), u(k))=x(k)+T f(x(k), u(k))
$$

in model predictive control of a continuous-time plant $\dot{x}=f(x, u)$. While this approach is very attractive to use because of the reduced computational effort in obtaining the approximate discrete-time model $F_{T}^{a}$, we show below that it may have serious limitations.

Note that for $T=h$ we need to use Theorem 4.5 which (among other things) requires:

C1. A lower bound on the optimal value function that is uniform in small $T$, that is there exist $T^{*}>0$ and $\sigma_{1} \in \mathcal{K}_{\infty}$ such that

$$
\sigma_{1}(\|x\|) \leq W_{T}^{a}(x), \forall x, T \in\left(0, T^{*}\right) .
$$

C2. Boundedness of the optimal controller $u_{T}^{a, *}$ on compact sets uniform in small $T$, that is for any $\Delta>0$ there exists $T^{*}>0$ and $M>0$ such that for all $\|x\| \leq M, T \in\left(0, T^{*}\right)$ :

$$
\left\|u_{T}^{a, *}(x)\right\| \leq M
$$

C3. $W_{T}^{a}$ locally Lipschitz, uniformly in small $T$, that is (4.6) holds.

It is well known from optimal control theory that even for fixed $T>0$ one can not expect $W_{T}^{a}$ to be locally Lipschitz in general and hence condition C3 usually does not hold. Moreover, note that the inequalities (5.6) and (5.12) seem to suggest that in general for any fixed $x$ we may have that $W_{T}^{a}(x) \rightarrow 0$ and $\left\|u_{T}^{a, *}(x)\right\| \rightarrow \infty$ as $T \rightarrow 0$, which violates conditions $\mathrm{C} 1$ and $\mathrm{C} 2$. The next example shows that this can indeed happen when $T=h$. 
Example 5.8 Consider the scalar system

$$
\dot{x}=u^{3}
$$

with $u \in U=\mathbb{R}$ and the running cost $l(x, u)=\|x\|^{2}+\|u\|^{2}$. The corresponding exact discrete-time model is given by

$$
x(k+1)=x(k)+T u^{3}(k)=: F_{T}(x(k), u(k)),
$$

so, the control sequence $\bar{u}$ induced by the state feedback law

$$
u_{T}^{*}(x)=-(x / T)^{1 / 3}
$$

yields

$$
\sum_{k=0}^{\infty} T l\left(\phi_{T}(k, x(0), \bar{u}), u(k)\right)=T\left(\|x(0)\|^{2}+(x(0) / T)^{2 / 3}\right)=T\|x(0)\|^{2}+T^{1 / 3}\|x(0)\|^{2 / 3} .
$$

Consequently, we obtain

$$
W_{T}(x(0)) \leq T\|x(0)\|^{2}+T^{1 / 3}\|x(0)\|^{2 / 3} .
$$

Setting $W_{T}(x(0))=T\|x(0)\|^{2}+T^{1 / 3}\|x(0)\|^{2 / 3}$ one sees that the equality

$$
l\left(x, u_{T}^{*}(x)\right)+W_{T}\left(F_{T}\left(x, u_{T}^{*}(x)\right)=\inf _{u \in U}\left\{l(x, u)+W_{T}\left(F_{T}(x, u)\right\}\right.\right.
$$

holds (one verifies that for all $x, T$ the term on the right hand side has only two local minima located at $u=0$ and $u=u_{T}^{*}(x)$ and the latter yields a smaller value). Hence, the feedback law $u_{T}^{*}(x)$ is optimal for this problem.

Note that for any fixed $x \neq 0$ we have $T \rightarrow 0 \Longrightarrow W_{T}(x) \rightarrow 0$ and $\left\|u_{T}^{*}(x)\right\| \rightarrow \infty$.

While in the example discussed above $u_{T}^{*}(x)$ still asymptotically stabilizes the exact model (due to the fact that for this simple system the exact discrete-time model and its Euler approximation coincide), in general this phenomenon poses a serious problem and $u_{T}^{a, *}$ may in general destabilize the family $F_{T}^{e}$. Several examples illustrating this phenomenon can be found in [17].

As a result of the above discussion, it is obvious that one can either search for conditions on $f, F_{T}^{a}$ and $l_{T}$ to guarantee that $\mathrm{C} 1, \mathrm{C} 2$ and $\mathrm{C} 3$ hold, or simply assume that they hold. While it is apparent that the first approach poses interesting and relevant questions, we did not pursue it in this paper. Using the second approach we can state Theorem 6.2. Before we state the theorem we need to restate the definition of asymptotic controllability:

Definition 5.9 Let $\beta \in \mathcal{K} \mathcal{L}$ be given. The family of systems $x(k+1)=F_{T}(x(k), u(k))$ is called semiglobally asymptotically controllable to the origin with vanishing controls if for each $\Delta>0$ there exists $T^{*}>0$ such that for all $T \in\left(0, T^{*}\right]$ and each $x \in \bar{B}_{\Delta}$ there exists $\bar{u} \in \mathcal{U}$ such that

$$
\left\|\phi_{T}(k, x, \bar{u})\right\|+\|u(k)\| \leq \beta(\|x\|, T k) .
$$


Theorem 5.10 Let $T=h$. Let $\beta \in \mathcal{K} \mathcal{L}$ and $l_{T}(\cdot, \cdot)$ satisfying Assumption 5.1 be given. Let $\beta$ generate $\alpha_{1}, \alpha_{2} \in \mathcal{K}_{\infty}$ using Proposition 5.3 and let $l_{T}$ generate $\rho_{1}, \rho_{2} \in \mathcal{K}_{\infty}$ via (5.2). Suppose that:

(i) The family of approximate models $F_{T}^{a}$ satisfies: for any $\Delta>0$ there exist $N>0$ and $T^{*}>0$ such that for all $T \in\left(0, T^{*}\right]$ and $x \in \bar{B}_{\Delta}$ we have:

$$
\left\|F_{T}^{a}(x, u)-F_{T}^{a}(y, u)\right\| \leq e^{N T}\|x-y\| .
$$

(ii) The family of approximate models $F_{T}^{a}$ is semiglobally asymptotically controllable to the origin with vanishing controls.

(iii) There exists $C>0$ such that the condition (5.4) holds.

(iv) There exists $\sigma_{1} \in \mathcal{K}_{\infty}$ and $T^{*}>0$ such that for all $x$ and $T \in\left(0, T^{*}\right]$ we have

$$
\sigma_{1}(\|x\|) \leq W_{T}^{a}(x) .
$$

(v) For any $\Delta>0$ there exist $T^{*}>0$ and $L>0$ such that

$$
\left|W_{T}^{a}(x)-W_{T}^{a}(y)\right| \leq L\|x-y\|,
$$

for all $x, y \in \bar{B}_{\Delta}, T \in\left(0, T^{*}\right]$.

Then, for the family of systems $F_{T}^{a}$ there exists a solution to the family of optimal control problems:

$$
\min _{\bar{u} \in \mathcal{U}} \sum_{k=0}^{\infty} T l_{T}\left(\phi_{T}^{a}(k, x, \bar{u}), u(k)\right),
$$

of the form

$$
u(k)=u_{T}^{a, *}(x(k))
$$

such that:

(i') The family $\left(F_{T}^{a}, u_{T}^{a, *}\right)$ is semiglobally practically stable with a Lipschitz Lyapunov function.

Suppose, moreover, that the additional condition holds:

(ii') The family of approximate models $F_{T}^{a}$ is semiglobally consistent with $F_{T}^{e}$.

(iii') The family of controllers $u_{T}^{a, *}$ is semiglobally uniformly bounded.

Then, there exists $\beta_{1} \in \mathcal{K} \mathcal{L}$ such that for any strictly positive $\left(D_{1}, \delta\right)$ there exists $T^{*}>0$ such that for all $x_{\circ} \in B_{D_{1}}$ and all $T \in\left(0, T^{*}\right]$, the solutions of the family $\left(F_{T}^{e}, u_{T}^{a, *}\right)$ satisfy:

$$
\left\|\phi_{T}^{e}\left(k, x_{\circ}\right)\right\| \leq \beta_{1}\left(\left\|x_{\circ}\right\|, k T\right)+\delta, \quad \forall k \in \mathbb{N}_{0} .
$$


Proof: We provide only a sketch of the proof since it is very similar to the proof of Theorem 5.4. The only thing to prove is that (i)-(v) imply (i') since the rest of the proof follows immediately from Theorem 4.5.

Note that the condition (v) implies (4.6) and the condition (iv) implies the lower bound in (4.3). The upper bound in (4.3) is established in the same way as that in the proof of Theorem 5.4. The inequality (4.4) is established in the same way as in the proof of Theorem 5.4, which completes the proof.

\section{Finite horizon with terminal cost problems}

In practice, the optimal control problem under consideration will often not be solved over an infinite time horizon, but using a suitable terminal cost. There are various ways to introduce a terminal cost, see, e.g., [5, Sections III.3 and IV.3], and we believe that our approach can be adjusted in order to cope with most of them. In order to illustrate this procedure, we consider the special type of terminal cost introduced by Kreisselmeier and Birkhölzer in [13].

We introduce a family of continuous and positive definite functions $\bar{W}_{T, h}: \mathbb{R}^{n} \rightarrow \mathbb{R}_{0}^{+}$for $T \in\left(0, T^{*}\right]$ and $h \in(0, T]$ and consider the family of finite horizon optimal control problems with terminal costs

$$
W_{T, h}^{a}(x):=\inf _{\bar{u} \in \mathcal{U}, k^{\prime} \in \mathbb{N}_{0}}\left\{\sum_{k=0}^{k^{\prime}-1} T l_{h}\left(\phi_{T, h}^{a}(k, x, \bar{u}), u(k)\right)+\bar{W}_{T, h}\left(\phi_{T, h}^{a}\left(k^{\prime}, x, \bar{u}\right)\right)\right\} .
$$

Using our continuity assumptions on $F_{T, h}^{a}$ and $l_{h}$ in $u$ it is easily seen that there always exists a feedback law $u_{T, h}^{a, *}: \mathbb{R}^{n} \rightarrow U$ satisfying

$$
T l_{h}\left(x, u_{T, h}^{a, *}(x)\right)+W_{T, h}^{a}\left(F_{T, h}^{a}\left(x, u_{T, h}^{a, *}(x)\right)\right)=\min _{u \in U}\left\{T l_{h}(x, u)+W_{T, h}^{a}\left(F_{T, h}^{a}(x, u)\right)\right\} .
$$

Moreover, observe that using (6.2) the dynamic programming equation for $W_{T, h}^{a}(x)$ reads

$$
W_{T, h}^{a}(x)=\min \left\{T l_{h}\left(x, u_{T, h}^{a, *}(x)\right)+W_{T, h}^{a}\left(F_{T, h}^{a}\left(x, u_{T, h}^{a, *}(x)\right)\right), \bar{W}_{T, h}(x)\right\} .
$$

\subsection{Stabilization with fixed sampling rate $T$}

In this section we consider $T$ as an arbitrary but fixed positive sampling rate. In order to derive a stabilization result we need the following assumption on $\bar{W}_{T, h}$.

Assumption 6.1 The following hold:

(i) $\bar{W}_{T, h}$ is continuous, uniformly in small $h$.

(ii) There exist $h^{*}>0$ and two class $\mathcal{K}_{\infty}$ functions $\gamma_{1}$ and $\gamma_{2}$ such that the inequality

$$
\gamma_{1}(\|x\|) \leq \bar{W}_{T, h}(x) \leq \gamma_{2}(\|x\|)
$$

holds for all $x$ and $h \in\left(0, h^{*}\right]$. 
Theorem 6.2 Let strictly positive real numbers $(\Delta, T)$ and the family of functions $\bar{W}_{T, h}(\cdot)$ satisfying Assumption 6.1 and the family of functions $l_{h}(\cdot, \cdot)$ satisfying Assumption 5.1 be given.

Suppose that:

(i) The family of approximate models $F_{T, h}^{a}$ satisfies Assumption 3.2.

(ii) For any $d>0$ there exists $h^{*}>0$ such that for all $h \in\left(0, h^{*}\right]$ there exists a solution to the optimization problem (6.1) that satisfies

$$
W_{T, h}^{a}(x)<\bar{W}_{T, h}(x) \quad \forall x \in B_{\Delta}-B_{d}, h \in\left(0, h^{*}\right] .
$$

Then there exists $M>0$ such that $u_{T, h}^{a, *}(\cdot)$ from (6.2) satisfies the following properties for $D=\Delta$ :

(i') The family of controllers $u_{T, h}^{a, *}$ is $(T, D, M)$-uniformly bounded.

(ii') The family $\left(F_{T, h}^{a}, u_{T, h}^{a, *}\right)$ is $(T, D)$-stable with a continuous Lyapunov function.

Suppose, moreover, that the additional condition holds:

(iii') The family of approximate models $F_{T, h}^{a}$ is $(T, D, M)$-consistent with $F_{T, h}^{e}$.

Then, there exists $D_{1} \in(0, D)$ and $\beta_{1} \in \mathcal{K} \mathcal{L}$ and for any $\delta>0$ there exists $h^{*}>0$ such that for all $x_{\circ} \in \bar{B}_{D_{1}}$ and all $h \in\left(0, h^{*}\right]$, the solutions of the family $\left(F_{T, h}^{e}, u_{T, h}^{a, *}\right)$ satisfy:

$$
\left\|\phi_{T, h}^{e}\left(k, x_{\circ}\right)\right\| \leq \beta_{1}\left(\left\|x_{\circ}\right\|, k T\right)+\delta, \quad \forall k \in \mathbb{N}_{0} .
$$

Proof: Similar to the proof of Theorem 5.4, the main task is to prove that conditions (i), (ii) and (iii) imply conditions (i') and (ii'). Then, again, the last statement follows immediately from (i'), (ii') and (iii') via Theorem 4.4.

(i) $+($ ii $)+($ iii $) \Rightarrow($ ii')

We use the optimal value function $W_{T, h}^{a}(x)$ as a Lyapunov function candidate and verify the conditions (4.3), (4.4) and (4.5) of Definition 4.3 for the family $\left(F_{T, h}^{a}, u_{T, h}^{a, *}\right)$.

Let Assumption 6.1 generate $h_{1}^{*}>0$ and $\gamma_{1}, \gamma_{2} \in \mathcal{K}_{\infty}$. Let Assumption 5.1 generate $h_{2}^{*}>0$ and $\rho_{1}, \rho_{2} \in \mathcal{K}_{\infty}$. Let $(T, \Delta)$ come from conditions of the theorem. Define $D:=\Delta$ and let $\delta_{1}$ be arbitrary strictly positive real number ${ }^{2}$. Let $d$ be such that

$$
T \rho_{2}(d)+\gamma_{2}\left(e^{L T} d\right) \leq T \delta_{1} .
$$

Let $(D, d)$ generate $h_{3}^{*}>0$ using condition (ii) of the theorem. Let $h^{*}:=\min \left\{h_{1}^{*}, h_{2}^{*}, h_{3}^{*}\right\}$. In the rest of the proof we consider arbitrary $x \in \bar{B}_{D}$ and $h \in\left(0, h^{*}\right]$.

\footnotetext{
${ }^{2}$ Like in the previous section, we prove that all conditions of Definition 4.3 hold with $\delta_{2}=0$.
} 
First we prove that (4.3) holds. Using definition of $W_{T, h}^{a}$, we obtain the inequality

$$
W_{T, h}^{a}(x) \leq \bar{W}_{T, h}(x) \leq \gamma_{2}(\|x\|)=: \sigma_{2}(\|x\|) .
$$

For the lower bound, observe from (6.3) that we either have

$$
W_{T, h}^{a}(x)=\bar{W}_{T, h}(x) \geq \gamma_{1}(\|x\|)
$$

or

$$
W_{T, h}^{a}(x) \geq T l_{h}\left(x, u_{T, h}^{a, *}(x)\right) \geq T \rho_{1}(\|x\|)
$$

and hence

$$
W_{T, h}^{a}(x) \geq \min \left\{\gamma_{1}(\|x\|), T \rho_{1}(\|x\|)\right\}=: \sigma_{1}(\|x\|),
$$

which completes the proof of (4.3).

Next we show (4.4) for the family $\left(F_{T, h}^{a, *}, u_{T, h}^{a, *}\right)$. From our choice of $x$ and $h$, for any $x \in B_{D}-B_{d}$ we obtain that the "min" in (6.3) is attained in the first term, hence

$$
W_{T, h}^{a}\left(F_{T, h}^{a}\left(x, u_{T, h}^{a, *}(x)\right)\right)-W_{T, h}(x)=-T l_{h}\left(x, u_{T, h}^{a, *}(x)\right) .
$$

For $x \in B_{d}$ observe that inequality $(3.7)$ and $F_{T, h}^{a}(0,0)=0$ imply $\left\|F_{T, h}^{a}(x, 0)\right\| \leq e^{L T}\|x\|$. Hence from (6.2) we obtain

$$
\begin{aligned}
T l_{h}\left(x, u_{T, h}^{a, *}(x)\right)+W_{T, h}^{a}\left(F_{T, h}^{a}\left(x, u_{T, h}^{a, *}(x)\right)\right) & \leq T l_{h}(x, 0)+W_{T, h}^{a}\left(F_{T, h}^{a}(x, 0)\right) \\
& \leq T \rho_{2}(\|x\|)+\gamma_{2}\left(e^{L T}\|x\|\right) \\
& \leq T \rho_{2}(d)+\gamma_{2}\left(e^{L T} d\right) \\
& \leq T \delta_{1} .
\end{aligned}
$$

Since $W_{T, h}^{a}(x) \geq 0$ this implies

$$
W_{T, h}^{a}\left(F_{T, h}^{a}\left(x, u_{T, h}^{a, *}(x)\right)\right)-W_{T, h}^{a}(x) \leq-T l_{h}\left(x, u_{T, h}^{a, *}(x)\right)+T \delta_{1} .
$$

Then for $x \in B_{D}$ either (6.6) or (6.8) holds, which implies

$$
\begin{aligned}
W_{T, h}^{a}\left(F_{T, h}^{a}\left(x, u_{T, h}^{a, *}(x)\right)\right)-W_{T, h}^{a}(x) & \leq-T l_{h}\left(x, u_{T, h}^{a, *}(x)\right)+T \delta_{1} \\
& \leq-T \rho_{1}(\|x\|)+T \delta_{1} \\
& =:-T \sigma_{3}(\|x\|)+T \delta_{1},
\end{aligned}
$$

i.e., the desired estimate (4.4) holds.

In order to show the continuity property (4.5), first observe that by the continuity condition on $\bar{W}_{T, h}$ from Assumption 6.1 for any given $\tilde{\delta}>0$ we find $\tilde{c}>0$ such that for all $x, y \in B_{D}$ with $\|x-y\| \leq \tilde{c}$ we obtain

$$
\left|\bar{W}_{T, h}^{a}(x)-\bar{W}_{T, h}^{a}(y)\right| \leq \tilde{\delta} .
$$

Consider the (arbitrary) $\delta_{1}>0$, which has been chosen above. Then for any $x \in B_{D}$ we find a control sequence $\bar{u}$ and a value $\bar{k} \in \mathbb{N}_{0}$ such that

$$
W_{T, h}^{a}(x)+\delta_{1} / 4 \geq \sum_{k=0}^{\bar{k}-1} T l_{h}\left(\phi_{T, h}^{a}(k, x, \bar{u}), u(k)\right)+\bar{W}_{T, h}\left(\phi_{T, h}^{a}(\bar{k}, x, \bar{u})\right) .
$$


Proceeding similar to the proof of Theorem 5.4, we find a constant $c>0$ and, from Lemma 8.2 , a time $\tau>0$ such that either $\bar{k}-1 \leq \tau / T$ or for any $y \in B_{D}$ with $\|x-y\| \leq c$ the inequality

$$
W_{T, h}^{a}\left(\phi_{T, h}^{a}(j, y, \bar{u})\right) \leq \delta_{1} / 8
$$

holds for some $j \in \mathbb{N}_{0}$ with $j \leq \tau / T$. If $\bar{k}-1>\tau / T$ holds we can exactly follow the proof of Theorem 5.4 to obtain that the implication

$$
\|x-y\| \leq c \Rightarrow W_{T, h}^{a}(y)-W_{T, h}^{a}(x) \leq \delta_{1}
$$

holds. Otherwise, i.e., when $\bar{k}-1 \leq \tau / T$, using the values defined in the proof of Theorem 5.4 , for any $y \in B_{D}$ with $\|x-y\| \leq c$ we obtain

$$
\begin{aligned}
W_{T, h}^{a}(y)-W_{T, h}^{a}(x) \leq & \sum_{k=0}^{\bar{k}-1} T l_{h}\left(\phi_{T, h}^{a}(k, y, \bar{u}), u(k)\right)+\bar{W}_{T, h}\left(\phi_{T, h}^{a}(\bar{k}, y, \bar{u})\right) \\
& \quad-\sum_{k=0}^{\bar{k}-1} T l_{h}\left(\phi_{T, h}^{a}(k, x, \bar{u}), u(k)\right)-\bar{W}_{T, h}\left(\phi_{T, h}^{a}(\bar{k}, x, \bar{u})\right)+\delta_{1} / 4 \\
\leq & \delta_{1} / 2+\delta_{1} / 4+\bar{W}_{T, h}\left(\phi_{T, h}^{a}(\bar{k}, y, \bar{u})\right)-\bar{W}_{T, h}\left(\phi_{T, h}^{a}(\bar{k}, x, \bar{u})\right) .
\end{aligned}
$$

Setting $\tilde{\delta}$ from (6.9) equal to $\delta_{1} / 4$ we find $\tilde{c}$ such that

$$
\bar{W}_{T, h}\left(\phi_{T, h}^{a}(\bar{k}, y, \bar{u})\right)-\bar{W}_{T, h}\left(\phi_{T, h}^{a}(\bar{k}, x, \bar{u})\right) \leq \delta_{1} / 4
$$

if $\left\|\phi_{T, h}^{a}(\bar{k}, x, \bar{u})-\phi_{T, h}^{a}(\bar{k}, y, \bar{u})\right\| \leq \tilde{c}$. Using that $\bar{k}-1 \leq \tau / T$ and that the control sequence $u_{T, h}^{a, *}$ is bounded by Lemma 8.1, by reducing $c>0$, if necessary, Lemma 8.4 guarantees that $\|x-y\| \leq c$ implies $\left\|\phi_{T, h}^{a}(\bar{k}, x, \bar{u})-\phi_{T, h}^{a}(\bar{k}, y, \bar{u})\right\| \leq \tilde{c}$. Thus, also in the case $\bar{k}-1 \leq \tau / T$ we obtain the implication

$$
\|x-y\| \leq c \Rightarrow W_{T, h}^{a}(y)-W_{T, h}^{a}(x) \leq \delta_{1} .
$$

Since the same estimate for $W_{T, h}^{a}(x)-W_{T, h}^{a}(y)$ follows by symmetry, this proves (4.5) and thus the proof of (ii').

(i) $+($ ii $)+($ iii $) \Rightarrow($ i')

For $x \in B_{D}-B_{d}$ this follows exactly as in the proof of Theorem 5.4 and we have that (5.12) holds. For $x \in B_{d}$ from inequality (6.7) we obtain

$$
T \rho_{1}\left(\left\|u_{T, h}^{a, *}(x)\right\|\right) \leq T l_{h}\left(x, u_{T, h}^{a, *}(x)\right) \leq T \rho_{2}(d)+\gamma_{2}\left(e^{L T} d\right),
$$

implying that for all $x \in B_{D}$ and $h \in\left(0, h^{*}\right]$ we have

$$
\left\|u_{T, h}^{a, *}(x)\right\| \leq \max \left\{\rho_{1}^{-1}\left(\frac{\sigma_{2}(D)}{T}\right), \rho_{1}^{-1}\left(\rho_{2}(d)+\frac{\gamma_{2}\left(e^{L T} d\right)}{T}\right)\right\}=: M
$$

which completes the proof. 


\subsection{Stabilization with varying sampling rate $T=h$}

We now state conditions that guarantee that optimization based stabilization of sampleddata systems via their approximate discrete-time models with $T=h$ can be successfully carried out. Consider the following version of Assumption 6.1 for $T=h$.

Assumption 6.3 There exists $T^{*}>0$ such that:

(i) $\bar{W}_{T}$ is continuous, uniformly in $T \in\left(0, T^{*}\right]$.

(ii) There exist two class $\mathcal{K}_{\infty}$ functions $\gamma_{1}$ and $\gamma_{2}$ such that the inequality

$$
\gamma_{1}(\|x\|) \leq \bar{W}_{T}(x) \leq \gamma_{2}(\|x\|)
$$

holds for all $x$ and $T \in\left(0, T^{*}\right]$.

Theorem 6.4 Let functions $\bar{W}_{T}(\cdot)$ satisfying Assumption 6.3 and $l_{T}(\cdot, \cdot)$ satisfying Assumption 5.1 be given.

Suppose that:

(i) The family of approximate models $F_{T}^{a}$ satisfies Assumption 3.2 and satisfies that for each $\Delta>0$ there exist $\gamma \in \mathcal{K}$ and $T^{*}>0$ such that the inequality

$$
\left\|F_{T}(x, 0)-x\right\| \leq T \gamma(\|x\|)
$$

holds for all $\|x\| \leq \Delta$ and all $T \in\left(0, T^{*}\right]$.

(ii) For any pair of positive real values $(D, d)$ there exists $T^{*}>0$ such that for all $T \in\left(0, T^{*}\right]$ there exists a solution to the optimization problem (6.1) that satisfies

$$
W_{T}^{a}(x)<\bar{W}_{T}(x) \quad \forall x \in B_{D}-B_{d}, T \in\left(0, T^{*}\right] .
$$

(iii) For any $\Delta>0$ there exist $L>0$ and $T^{*}>0$ such that

$$
\left|W_{T}^{a}(x)-W_{T}^{a}(y)\right| \leq L\|x-y\|,
$$

for all $x, y \in B_{\Delta}, T \in\left(0, T^{*}\right]$.

(iv) There exist $\sigma_{1} \in \mathcal{K}_{\infty}$ and $T^{*}>0$ such that for all $x$ and $T \in\left(0, T^{*}\right]$ we have

$$
\sigma_{1}(\|x\|) \leq W_{T}^{a}(x)
$$

Then we have that:

(i') The family $\left(F_{T}^{a}, u_{T}^{a, *}\right)$ is semiglobally stable with a Lipschitz Lyapunov function.

Suppose, moreover, that the additional condition holds: 
(ii') The family of approximate models $F_{T}^{a}$ is semiglobally consistent with $F_{T}^{e}$

(iii') The family of controllers $u_{T}^{a, *}$ is semiglobally uniformly bounded.

Then, there exists $\beta_{1} \in \mathcal{K} \mathcal{L}$ such that for any strictly positive $\left(D_{1}, \delta\right)$ there exists $T^{*}>0$ such that for all $x_{\circ} \in \bar{B}_{D_{1}}$ and all $T \in\left(0, T^{*}\right]$, the solutions of the family $\left(F_{T}^{e}, u_{T}^{a, *}\right)$ satisfy:

$$
\left\|\phi_{T}^{e}\left(k, x_{\circ}\right)\right\| \leq \beta_{1}\left(\left\|x_{\circ}\right\|, k T\right)+\delta, \quad \forall k \in \mathbb{N}_{0} .
$$

Proof: It suffices to prove that (i)-(iv) imply (i'), because then the statement follows from (i'), (ii') and (iii') by applying Theorem 4.5.

Since the condition (iii) implies (4.6), for proving (i') we only have to show (4.3) and (4.4).

Let $\left(D, \delta_{1}\right)$ be given ${ }^{3}$. Let $\bar{W}_{T}$ generate via Assumption 6.3 the functions $\gamma_{1}, \gamma_{2} \in \mathcal{K}_{\infty}$ and $T_{1}^{*}>0$. Let $l_{T}$ generate via Assumption 5.1 the functions $\rho_{1}, \rho_{2} \in \mathcal{K}_{\infty}$ and $T_{2}^{*}>0$. Let the condition (iv) generate $\sigma_{1}$ and $T_{3}^{*}>0$. Let $\Delta=D$ generate via the condition (i) the function $\gamma \in \mathcal{K}$ and $T_{4}^{*}>0$. Let $\Delta_{1}=D+\gamma(D)$ generate $L>0$ and $T_{5}^{*}>0$ via the condition (iii). Let $d>0$ be such that

$$
\rho_{2}(d)+L \gamma(d) \leq \delta_{1}
$$

Let $(D, d)$ generate $T_{6}^{*}>0$ via the condition (ii). Let $T^{*}=\min \left\{1, T_{1}^{*}, T_{2}^{*}, T_{3}^{*}, T_{4}^{*}, T_{5}^{*}, T_{6}^{*}\right\}$. Consider arbitrary $T \in\left(0, T^{*}\right]$ and $x \in \bar{B}_{D}$.

In order to prove (4.3), observe that the lower bound follows from the condition (iv) while the upper bound follows immediately from the inequality $W_{T}^{a}(x) \leq \bar{W}_{T}(x)$ and Assumption 6.3. Recall from (6.3) that the dynamic programming equation for $W_{T}^{a}(x)$ reads

$$
W_{T}^{a}(x)=\min \left\{T l_{T}\left(x, u_{T}^{a, *}(x)\right)+W_{T}^{a}\left(F_{T}^{a}\left(x, u_{T}^{a, *}(x)\right)\right), \bar{W}_{T}(x)\right\} .
$$

For all $T \in\left(0, T^{*}\right]$ and all $x \in \bar{B}_{D}-\bar{B}_{d}$ we obtain that the "min" is attained in the first term, hence

$$
W_{T}^{a}\left(F_{T}^{a}\left(x, u_{T}^{a, *}(x)\right)\right)-W_{T}^{a}(x)=-T l_{T}\left(x, u_{T}^{a, *}(x)\right) .
$$

For $x \in \bar{B}_{d}$ recall Assumption (i), which yields $\left\|F_{T, h}^{a}(x, 0)-x\right\| \leq T \gamma(\|x\|)$. Hence from (6.2) we obtain

$$
\begin{aligned}
T l_{T}\left(x, u_{T}^{a, *}(x)\right)+W_{T}^{a}\left(F_{T}^{a}\left(x, u_{T}^{a, *}(x)\right)\right)-W_{T}^{a}(x) & \leq T l_{T}(x, 0)+W_{T}^{a}\left(F_{T}^{a}(x, 0)\right)-W_{T}^{a}(x) \\
& \leq T \rho_{2}(\|x\|)+L T \gamma(\|x\|) \\
& \leq T\left(\rho_{2}(d)+L \gamma(d)\right) \leq T \delta_{1} .
\end{aligned}
$$

Hence, for $x \in \bar{B}_{D}$ either (6.12) or (6.13) holds, which implies

$$
W_{T}^{a}\left(F_{T}^{a}\left(x, u_{T}^{a, *}(x)\right)\right)-W_{T}^{a}(x) \leq-T l_{T}\left(x, u_{T}^{a, *}(x)\right)+T \delta_{1} \leq-T \rho_{1}(\|x\|)+T \delta_{1},
$$

i.e., the desired estimate (4.4) with $\alpha_{3}(r):=\rho_{1}(r)$.

\footnotetext{
${ }^{3}$ We will again prove (4.4) with $\delta_{2}=0$.
} 


\section{Conclusion and Outlook}

Results on optimization based stabilization of sampled-data systems via approximate discrete-time plant models are presented. Infinite horizon and finite horizon with terminal cost optimization problems were considered. In both cases it was shown under reasonable assumptions that when integration period $h$ is independent of the sampling period $T$, then one can use an approximate discrete-time plant model in the controller design to achieve stability of the exact discrete-time plant model. On the other hand, if $T=h$ then optimization based stabilization of sampled-data systems via approximate discretetime models requires much stronger assumptions to produce a stabilizing controller for the exact discrete-time plant model. Several examples are presented to illustrate the most common problems with this approach.

Apart from the optimal control problems we have considered in this paper, one of the most important optimal control based techniques used in nonlinear stabilization problems is receding horizon or model predictive control (RHC or MPC), cf. the references in the introduction. Due to the special structure of RHC and MPC techniques, our results in this paper are not directly applicable. We do, however, think that similar analysis techniques as we have used here can be applied also to these kind of controllers. Rigorous results in this direction are currently under investigation.

\section{Appendix}

Lemma 8.1 Let $l_{h}$ satisfy (5.2) with some $\rho_{1}, \rho_{2} \in \mathcal{K}_{\infty}$ and $h^{*}>0$. Then, for any strictly positive $(S, T), h \in\left(0, h^{*}\right]$ and $x \in \mathbb{R}^{n}, \bar{u} \in \mathcal{U}, \bar{k} \in \mathbb{N}_{0}$ satisfying

$$
\sum_{k=0}^{\bar{k}} T l_{h}\left(\phi_{T, h}^{a}(k, x, \bar{u}), u(k)\right) \leq S
$$

we have

$$
\left\|\phi_{T, h}^{a}(k, x, \bar{u})\right\|+\|u(k)\| \leq \rho_{1}^{-1}(S / T) \quad \forall k \in \mathbb{N}_{0}, k \leq \bar{k}
$$

Proof: Let (8.1) hold and assume the existence of $k \in \mathbb{N}_{0}$ with

$$
\left\|\phi_{T, h}^{a}(k, x, \bar{u})\right\|+\|u(k)\|>\rho_{1}^{-1}(S / T) .
$$

This implies using (5.2) that

$$
T l_{h}\left(\phi_{T, h}^{a}(k, x, \bar{u}), u(k)\right) \geq T \rho_{1}\left(\left\|\phi_{T, h}^{a}(k, x, \bar{u})\right\|+\|u(k)\|\right)>S,
$$

which contradicts (8.1).

Lemma 8.2 Let $l_{h}$ satisfy (5.2) with some $\rho_{1}, \rho_{2} \in \mathcal{K}_{\infty}$ and $h^{*}>0$. Then, for any pair of strictly positive numbers $(C, \varepsilon)$ there exists $\tau=\tau(C, \varepsilon)>0$ such that for any $x \in \mathbb{R}^{n}, \bar{u} \in \mathcal{U}$, 
any $T>0$ and $h \in\left(0, h^{*}\right]$ and any $\bar{k} \in \mathbb{N}$ with $\bar{k} T>\tau$ satisfying

$$
\sum_{k=0}^{\bar{k}} T l_{h}\left(\phi_{T, h}(k, x, \bar{u}), u(k)\right)<C
$$

there exists $j \in \mathbb{N}_{0}$ with $j \leq \tau / T$ such that $\left\|\phi_{T, h}^{a}(j, x, \bar{u})\right\|+\left\|u_{j}\right\| \leq \varepsilon$.

Proof: We define $\tau:=C / \rho_{1}(\varepsilon)$. Now assume $\left\|\phi_{T, h}^{a}(j, x, \bar{u})\right\|+\left\|u_{j}\right\| \geq \varepsilon$ for all $j \in \mathbb{N}_{0}$ with $j \leq \tau / T$. Denoting by $[\tau / T]$ the integer part of $\tau / T$ and using (5.2) we can conclude

$\sum_{k=0}^{\bar{k}} T l_{h}\left(\phi_{T, h}^{a}(k, x, \bar{u}), u(k)\right) \geq \sum_{k=0}^{[\tau / T]} T l_{h}\left(\phi_{T, h}^{a}(k, x, \bar{u}), u(k)\right) \geq[\tau / T+1] T \rho_{1}(\varepsilon) \geq \tau \rho_{1}(\varepsilon) \geq C$,

which contradicts (8.2).

The following lemma is a consequence of the consistency property. Similar results can be found in numerical analysis literature (see, for example [22, Theorems 6.2.1 and 6.2.2]) and the below given proof is provided for completeness.

Lemma 8.3 Let a 4 -tuple of strictly positive numbers $\left(\Delta_{1}, \Delta_{2}, T, \tau\right)$ be given and let $F_{T, h}^{a}$ be $\left(T, \Delta_{1}, \Delta_{2}\right)$-consistent with $F_{T, h}^{e}$. Let Assumption 3.2 hold. Consider any $x \in \mathbb{R}^{n}, \bar{u} \in \mathcal{U}$ satisfying

$$
k T \in[0, \tau] \Longrightarrow\left\|\phi_{T, h}^{a}(k, x, \bar{u})\right\| \leq \Delta_{1} \text { and }\|u(k)\| \leq \Delta_{2}
$$

Then, for any $\delta>0$ there exist strictly positive numbers $h^{*}=h^{*}\left(\Delta_{1}, \Delta_{2}, \delta, T\right)>0$ and $L=L\left(\Delta_{1}, \Delta_{2}, \delta\right)$ and $\gamma \in \mathcal{K}$ such that if $h \in\left(0, h^{*}\right]$ then $k T \in[0, \tau]$ implies that

$$
\left\|\phi_{T, h}^{e}(k, x, \bar{u})\right\| \leq \Delta_{1}+\delta
$$

and

$$
\left\|\phi_{T, h}^{a}(k, x, \bar{u})-\phi_{T, h}^{e}(k, x, \bar{u})\right\| \leq T \gamma(h) \frac{e^{L(\tau+T)}-1}{L T}
$$

Moreover, an analogous estimate holds if we exchange the roles of $\phi_{T, h}^{a}$ and $\phi_{T, h}^{e}$.

Proof: Let all conditions of the lemma hold. Let $L>0$ be the Lipschitz constant from (3.7) on the set $\bar{B}_{\max \left\{\Delta_{1}+\delta, \Delta_{2}\right\}}$ and let $\gamma \in \mathcal{K}$ come from the consistency property for the same set. Define

$$
h^{*}=\gamma^{-1}\left(\frac{\delta L}{e^{L(\tau+T)}-1}\right) .
$$

Consider now arbitrary $x, \bar{u}$ satisfying (8.3) and let $h \in\left(0, h^{*}\right]$. We abbreviate $x(k)^{a}=$ $\phi_{T, h}^{a}(k, x, \bar{u}), x(k)^{e}=\phi_{T}^{e}(k, x, \bar{u})$ and show the assertion by induction. For $k=0$ there is nothing to show. Pick $k \geq 1$ such that $k T \in[0, \tau]$ and assume for the purpose of induction that for the step $k-1$ the following holds

$$
\left\|\phi_{T, h}^{a}(k-1, x, \bar{u})-\phi_{T}^{e}(k-1, x, \bar{u})\right\| \leq \gamma(h) T \sum_{i=0}^{k-1} e^{L T i} \leq \delta .
$$


We can conclude using Assumption 3.2 and consistency that

$$
\begin{aligned}
& \left\|x(k)^{a}-x(k)^{e}\right\| \\
& =\left\|F_{T, h}^{a}\left(x_{k-1}^{a}, u_{k-1}\right)-F_{T, h}^{e}\left(x_{k-1}^{e}, u_{k-1}\right)\right\| \\
& \leq\left\|F_{T, h}^{a}\left(x_{k-1}^{a}, u_{k-1}\right)-F_{T, h}^{e}\left(x_{k-1}^{a}, u_{k-1}\right)\right\|+\left\|F_{T, h}^{e}\left(x_{k-1}^{a}, u_{k-1}\right)-F_{T, h}^{e}\left(x_{k-1}^{e}, u_{k-1}\right)\right\| \\
& \leq \gamma(h) T+e^{L T} \gamma(h) T \sum_{i=0}^{k-1} e^{L T i} \\
& \leq \gamma(h) T+\gamma(h) T \sum_{i=1}^{k} e^{L T i} \\
& \leq \gamma(h) T \sum_{i=0}^{k} e^{L T i}
\end{aligned}
$$

which shows that (8.6) holds for the step $k$. Finally, since $T \sum_{i=0}^{k} e^{L T i} \leq \frac{e^{L T(k+1)}-1}{L}$ and because of our choice of $h^{*}$, it follows that for all $h \in\left(0, h^{*}\right]$ and all $k T \in[0, \tau]$ we have

$$
\begin{aligned}
\left\|x(k)^{a}-x(k)^{e}\right\| & \leq T \gamma(h) \frac{e^{L(\tau+T)}-1}{L T} \\
& \leq \delta
\end{aligned}
$$

which proves that (8.5) holds. Finally, since $x_{k}^{a} \in \bar{B}_{\Delta_{1}}$ for all $k T \in[0, \tau]$ and (8.7) holds, this implies that (8.4) is satisfied, which completes the proof.

Lemma 8.4 Let arbitrary triple of strictly positive numbers $(\Delta, \tau, T)$ be given. Let $k_{0} \in \mathbb{N}$ be such that $k_{0} T \in[0, \tau]$. Let Assumption 3.2 hold for the family $F_{T, h}$. Then, for each $\delta>0$ there exists $c>0, L>0$ and $h^{*}>0$ such that for each $h \in\left(0, h^{*}\right]$ each two points $x, y \in \mathbb{R}^{n}$ with $\|x-y\| \leq c$ and each $\bar{u} \in \mathcal{U}$ satisfying

$$
\left\|\phi_{T, h}(k, x, \bar{u})\right\|+\|u(k)\| \leq \Delta \text { for all } k=0,1, \ldots, k_{0},
$$

the inequalities

$$
\left\|\phi_{T, h}(k, y, \bar{u})\right\| \leq \Delta+\delta
$$

and

$$
\left\|\phi_{T, h}(k, x, \bar{u})-\phi_{T, h}(k, y, \bar{u})\right\| \leq\|x-y\| e^{L T k} \leq \delta
$$

hold for all $k=0,1, \ldots, k_{0}$.

Proof: The proof follows with similar arguments as the proof for Lemma 8.3.

\section{References}

[1] M. Alamir and G. Bornard. On the stability of receding horizon control of nonlinear discrete-time systems. Syst. Contr. Lett., 23:291-296, 1994. 
[2] B. D. O. Anderson and J. Moore. Optimal control: linear quadratic methods. PrenticeHall, Engelwood Cliffs, 1990.

[3] D. Angeli and E. Mosca. Command governors for constrained nonlinear systems. IEEE Trans. Automat. Contr., 44:816-818, 1999.

[4] M. Arcak, D. Angeli, and E. Sontag. Stabilization of cascades using integral input-tostate stability. In Proceedings of the 40th IEEE Conference on Decision and Control, Orlando, Florida, 2001. To appear.

[5] M. Bardi and I. Capuzzo Dolcetta. Optimal Control and Viscosity Solutions of Hamilton-Jacobi-Bellman equations. Birkhäuser, Boston, 1997.

[6] G. de Nicolao, L. Magni, and R. Scattolini. Stabilizing receding-horizon control of nonlinear time-varying systems. IEEE Trans. Automat. Contr., 43:1030-1036, 1998.

[7] F. A. C. C. Fontes. A general framework to design stabilizing nonlinear model predictive controllers. Syst. Contr. Lett., 42:127-143, 2001.

[8] L. Grüne. Discrete feedback stabilization of semilinear control systems. ESAIM Control Optim. Calc. Var., 1:207-224, 1996.

[9] L. Grüne. Homogeneous state feedback stabilization of homogeneous systems. SIAM J. Control Optim., 38:1288-1314, 2000.

[10] A. Iserles. A First Course in the Numerical Analysis of Differential Equations. Cambridge Texts in Applied Mathematics. Cambridge University Press, 1995.

[11] S. S. Keerthy and E. G. Gilbert. An existence theorem for discrete-time infinite horizon optimal control problems. IEEE Trans. Automat. Contr., 30:907-909, 1985.

[12] S. S. Keerthy and E. G. Gilbert. Optimal infinite horizon feedback laws for a general class of constrained discrete-time systems: stability and moving horizon approximations. J. Optimiz. Theory Appl., 57:265-293, 1988.

[13] G. Kreisselmeier and T. Birkhölzer. Numerical nonlinear regulator design. IEEE Trans. Autom. Control, 39(1):33-46, 1994.

[14] D. Q. Mayne, J. B. Rawlings, C. V. Rao, and P. O. M. Scokaert. Constrained model predictive control: stability and optimality. Automatica, 36:789-814, 2000.

[15] D. Nešić, A. R. Teel, and P. V. Kokotović. Sufficient conditions for stabilization of sampled-data nonlinear systems via discrete-time approximations. Syst. Control Lett, 38:259-270, 1999.

[16] D. Nešić and A.R. Teel. Backstepping on the euler approximate model for stabilization of sampled-data nonlinear systems. In Conference on Decision and Control, pages 1737-1742, Orlando, 2001. IEEE.

[17] D. Nešić and A.R. Teel. A framework for stabilization of nonlinear sampled-data systems based on their approximate discrete-time models. IEEE Trans. Automat. Contr., 2002. Submitted for publication. 
[18] D. Nešić, A.R. Teel, and E.D. Sontag. Formulas relating KL stability estimates of discrete-time and sample-data nonlinear systems. System and Control Letters, 38:4960, 1999.

[19] E. D. Sontag. A Lyapunov like characterization of asymptotic controllability. SIAM J. Contr. Optim., 21:462-471, 1983.

[20] E. D. Sontag. Comments on integral variants of ISS. Syst. Contr. Lett., 34:93-100, 1998.

[21] J. Stoer and R. Bulirsch. Introduction to Numerical Analysis. Springer Verlag, New York, 1980.

[22] A. M. Stuart and A. R. Humphries. Dynamical Systems and Numerical Analysis. Cambridge University Press, 1996. 